\title{
Meditation in cardiac rehabilitation: Should we be thinking about it?
}

\author{
Raymond J. Gibbons, MD, MSc, ${ }^{\text {a }}$ and Randal J. Thomas, MD, MS ${ }^{a}$ \\ a Department of Cardiovascular Medicine, Mayo Clinic, Rochester, MN
}

Received Sep 27, 2019; accepted Sep 27, 2019

doi:10.1007/s12350-019-01920-8

\section{See related article, pp. 1596-1607}

Cardiac rehabilitation (CR) is a well-established component of cardiovascular care. It is a multidisciplinary, team-based delivery system for secondary CVD prevention that has proven, cost-effective benefits in a variety of patients with known cardiovascular disease, such as those who have experienced myocardial infarction, percutaneous coronary intervention (PCI), or coronary artery bypass surgery (CABG), or those with chronic stable angina. For this reason, CR is a Class 1 recommendation in clinical practice guidelines for these conditions; it is approved for reimbursement by the Center for Medicaid and Medicare Services (CMMS) for such patients. Unfortunately, $\mathrm{CR}$ is significantly underutilized in the USA and around the world. ${ }^{1-3}$ Current delivery models for CR need to be improved to assure that all eligible patients can receive the benefits of CR.

Is there room for improvement in the components and interventions included in CR?

This possibility is raised in the current issue of the Journal of Nuclear Cardiology in a paper by Bokhari et al. ${ }^{4}$ that reports a pilot study of the inclusion of meditation in CR. The study randomized a total of 56 African-Americans with coronary heart disease (CHD) to $\mathrm{CR}$ with and without transcendental meditation (TM). The study collected data on CHD risk factors and assessed myocardial flow reserve (MFR) by $\mathrm{N}-13$ ammonia positron emission tomography (PET) at baseline and after 12 weeks. The authors conclude that TM

Reprint requests: Raymond J. Gibbons, MD, MSc, Department of Cardiovascular Medicine, Mayo Clinic, Rochester, MN; gibbons.raymond@mayo.edu

J Nucl Cardiol 2021;28:1608-10.

1071-3581/\$34.00

Copyright (C) 2019 American Society of Nuclear Cardiology. may improve MFR in African-American patients with CHD; they suggest that their data be considered as preliminary pilot data, to help in the design of future, larger randomized clinical trials to better establish the benefit of TM.

The strengths of this study are obvious. It is highly novel, and focused on African-Americans in an attempt to reduce the widely recognized inequalities in health care. The investigators appear to have considerable expertise in TM. The use of TM is described in sufficient detail. The end-point of MFR by N-13 ammonia PET is well validated in the nuclear cardiology literature. ${ }^{5}$

However, the study has weaknesses that limit our ability to interpret the results. The first is its overall design and execution. It was carried out in two separate phases. In Phase I, 32 patients were randomly assigned to CR with or without TM. In Phase II, an additional 24 patients were randomly assigned to usual care (without $\mathrm{CR}$ ) with or without TM. "For simplicity and ease of presentation", 4 groups (CR vs no CR, TM vs no TM) were collapsed into 2 groups-with and without TM. Unfortunately, only 53 patients completed the baseline measurement of blood flow, only 43 patients completed the measurement of resting myocardial blood flow at 12 weeks, and only 37 patients (66\%) completed MFR assessment at 12 weeks. No comparison is provided of the one-third of the patients who did not complete the endpoint measurements with those who did. The results are therefore subject to an uncertain amount of ascertainment bias.

Since the authors reported on the effect of both CR and TM, a more conventional approach would have been a $2 \times 2$ factorial design that would have enrolled all of the 56 patients in a single randomized study, ${ }^{6}$ rather than two-phases. A $2 \times 2$ factorial design balances baseline variables across all of the patients, and has approximately equal sample sizes in all four groups. In contrast, the authors had smaller numbers of patients randomized in Phase II. Therefore, we cannot be certain that the baseline characteristics provided in Table 1 between 
patients with TM and without TM are balanced by randomization or by chance.

In addition to the missing endpoint measurements, the data regarding attendance suggests sub-optimal compliance. Attendance at the $\mathrm{CR}$ sessions was only about $60 \%$ overall. Of the patients assigned to TM, $71 \%$ completed instruction in the technique and 58\% attended all of the classes. Thus, the data that are presented may represent an underestimate of the true effects of both CR and TM, because of non-compliance.

The study did not control for the increased "contact time" in the TM group. Patients in the TM group appear to have spent at least 15 more hours with the staff. It is conceivable that the increased staff time gave the patients more time to ask questions, and understand their disease. They may have then complied better with medications, improving their risk factors and MFR. The measured benefits may reflect more staff contact time, rather than TM.

The statistical analysis was not conventional. As indicated in the Abstract, "based on guidelines for analysis of small pilot studies, data was analyzed for effect size (ES)." The reference supplied in the text ${ }^{7}$ supporting this approach is not a guideline; it is a commentary by two authors on a separate publication from the American Statistical Association (ASA). ${ }^{8}$ The commentary has a brief section on " $P$ values and small sample sizes" which states "depending on how small the study, a descriptive study with standardized differences may be more appropriate than an inferential approach with P values". In contrast, the ASA statement does not make any such recommendation regarding the analysis of small studies. There is a substantial statistical literature on the subject of effect size that is well beyond the scope of this editorial. Statistical texts routinely indicate that the power to detect a treatment effect depends on the size of the effect, the variability in the population and the sample sizes of the treatment groups. ${ }^{9}$ The ASA statement indicates that $\mathrm{p}$ values do not measure the size of the effect, or the importance of the result. Any effect, no matter how small, can produce an impressive $P$ value if the sample size and measurement precision are both high. Many of the publications on this topic try to distinguish statistical significance from clinical significance in the analysis of large clinical trials. $^{10}$

Because of the importance of effect size in the calculation of sample size and the frequent situation where data are too incomplete to estimate effect size, there are entire projects devoted to this issue. ${ }^{11}$ Any pilot data from small studies is therefore potentially useful for the estimation of effect size and sample size. We agree with the authors that the data on effect size presented in this manuscript may assist in the design of future trials. However, it will have to be interpreted in light of the study weaknesses of excess dropouts, noncompliance, uncontrolled staff time, and possible imbalance in randomization. Most importantly, by conventional analysis, this small study failed to show that TM had a statistically significant effect on $\operatorname{MFR}(\mathrm{p}=0.29)$.

Despite these limitations, the question raised by the study is still pertinent and important. Can CR be more effective if new methods of psychological health and healing are included? Interventions to identify and treat psychological stress and dysfunction are currently included as important parts of the core components of multidisciplinary CR services. In fact, multiple studies have identified the positive impact of CR on psychological health outcomes in patients with CVD. Exercise training, a key component of CR, has itself been shown to have positive effects on mood, depressive symptoms, and quality of life, thought to be due a number of potential mechanisms, including the production of endorphins and enkephalins, peptides that act as natural painkillers and mood elevators. ${ }^{12}$ Exercise training produces a number of other beneficial effects on health, such as improvements in functional capacity, cardiovascular symptom control, and myocardial blood flow. ${ }^{13}$

The psychological impact of CR likely goes beyond the impact of exercise training on psychological health. Other components of CR are likely to be intertwined, including the counseling that patients receive on stress management, nutrition, lifestyle change, disease management, and medication adherence. In addition, the effects of socialization and group support are also likely to help. Patients in CR programs typically undergo screening for stress, anxiety, and depression, and are referred to mental health care providers as needed.

Would the addition of TM to CR provide incremental improvement in psychological and physiological patient outcomes? Based on the paper by Bokhari and colleagues, we are unable to say. Additional research may be helpful in clarifying this issue further, but given the significant focus of $\mathrm{CR}$ on psychological health interventions and outcomes, it is likely that any incremental contributions of TM to current CR outcomes will be modest in degree, at most, and complimentary to current $\mathrm{CR}$ intervention components.

Medicine should always be interested in quality improvement and searching for how to do things better. We should therefore be thinking about TM, but only for future research studies. The data from this small pilot study does not justify adding TM to existing CR programs, until more evidence is available. The investigators might consider conducting one-year follow-up of their patients to ascertain their compliance with cardiac medications and their blood pressure 
control. Long-term compliance with proven cardiac medications remains a challenge. ${ }^{14,15}$ Current control of hypertension, particularly in African-Americans, is suboptimal. ${ }^{16}$ Any data suggesting improvement in these areas with TM would strongly encourage future research.

The current primary challenges for $\mathrm{CR}$ are systematic barriers to accessibility and uptake. Solutions to these barriers exist, but are not universally adopted. Unfortunately, one missed opportunity to strengthen CR utilization occurred with the bundled payment initiative announced by CMMS in 2018 that did not include any quality metrics for CR. Since costs were measured for 90 days, without any quality metrics beyond 30 days, after the myocardial infarction, PCI or CABG, the bundled payment program created an economic disincentive, to continue or expand CR programs. ${ }^{17}$ The inclusion of a single $\mathrm{CR}$ metric in the recently updated bundled payment program for 2020 is a step in the right direction. ${ }^{18}$ However, it may not be sufficient to prevent hospital administrators from discontinuing or restricting CR programs in their efforts to reduce 90-day costs. The overall pressure on hospitals to reduce costs will continue and potentially increase. We urge healthcare providers who recognize the value of $\mathrm{CR}$ to communicate its value to their hospital administrators. In addition, we urge providers with patients who have been satisfied and improved by $\mathrm{CR}$ to encourage these patients to communicate their feelings to their hospital administrators. These efforts are very important to offset the unfortunate economic disincentives. Healthcare providers and economists agree that the healthcare system must increase "value" in order to improve quality over the long-term ${ }^{19-21} \mathrm{CR}$, which decreases cost (from a societal perspective-not a hospital perspective) and improves outcomes, is a very highvalue program, but currently underutilized. We must do everything possible to reverse this adverse trend.

\section{Disclosure}

R.J. Gibbons and R.J. Thomas have nothing to disclose.

\section{References}

1. Turk-Adawi K, Supervia M, Lopez-Jimenez F, Pesah E, Ding R, Britto RR, et al. Cardiac rehabilitation availability and density around the globe. EClinicalMedicine. 2019;13:31-45.

2. van Engen-Verheul M, de Vries H, Kemps H, Kraaijenhagen R, de Keizer N, Peek N. Cardiac rehabilitation uptake and its determinants in the Netherlands. Eur J Prev Cardiol. 2013;20:349-56.

3. Beatty AL, Truong M, Schopfer DW, Shen H, Bachmann JM, Whooley MA. Geographic variation in cardiac rehabilitation participation in medicare and veterans affairs populations: Opportunity for improvement. Circulation. 2018;137:1899-908.
4. Bokhari S, Schneider RH, Salerno JW, Rainforth MV, GaylordKing C, Nidich SI. Effects of cardiac rehabilitation with and without meditation on myocardial blood flow using quantitative positron emission tomography: A pilot study. J Nucl Cardiol. 2019. https://doi.org/10.1007/s12350-019-01884-9.

5. Murthy VL, Bateman TM, Beanlands RS, Berman DS, BorgesNeto S, Chareonthaitawee P, et al. Clinical quantification of myocardial blood flow using PET: Joint position paper of the SNMMI Cardiovascular Council and the ASNC. J Nucl Med. 2018;59:273-93.

6. Yusuf S, Lonn E, Pais P, Bosch J, López-Jaramillo P, Zhu J, et al. Blood-pressure and cholesterol lowering in persons without cardiovascular disease. N Engl J Med. 2016;374:2032-43.

7. Tijssen JGP, Kolm P. Demystifying the new statistical recommendations: The Use and reporting of $\mathrm{p}$ values. J Am Coll Cardiol. 2016;68:231-3.

8. Wasserstein RL, Lazar NA. The ASA statement on p-values: Context, process, and purpose. Am Stat. 2016;70:129-33.

9. Glantz SA. Primer of biostatistics. 4th ed. New York: McGrawHill; 1997.

10. Diamond GA, Kaul S. On reporting of effect size in randomized clinical trials. Am J Cardiol. 2013;111:613-7.

11. Cook JA, Julious SA, Sones W, Hampson LV, Hewitt C, Berlin JA, et al. DELTA(2) guidance on choosing the target difference and undertaking and reporting the sample size calculation for a randomised controlled trial. Trials. 2018;19:606.

12. Blumenthal JA, Sherwood A, Babyak MA, Watkins LL, Smith PJ, Hoffman BM, et al. Exercise and pharmacological treatment of depressive symptoms in patients with coronary heart disease: results from the UPBEAT (Understanding the Prognostic Benefits of Exercise and Antidepressant Therapy) study. J Am Coll Cardiol. 2012;60:1053-63.

13. Yoshinaga K, Beanlands RSB, deKemp RA, Lortie M, Morin J, Aung M, et al. Effect of exercise training on myocardial blood flow in patients with stable coronary artery disease. Am Heart J. 2006;151:1324.e11-8.

14. Gibbons RJ, Miller TD. Optimal medical therapy for known coronary artery disease: A review. JAMA Cardiol. 2017;2:1030-5.

15. Shah ND, Dunlay SM, Ting HH, Montori VM, Thomas RJ, Wagie $\mathrm{AE}$, et al. Long-term medication adherence after myocardial infarction: Experience of a community. Am J Med. 2009;122:961.e7-13.

16. Ferdinand KC, Senatore FF, Clayton-Jeter H, Cryer DR, Lewin JC, Nasser SA, et al. Improving Medication adherence in cardiometabolic disease: Practical and regulatory implications. J Am Coll Cardiol. 2017;69:437-51.

17. Gibbons RJ. Doctor: Medicare's cuts to cardiac rehab programs will cost lives. http://www.startribunecom/doctor-medicare-s-cutsto-cardiac-rehab-programs-will-cost-lives/498068981/; 2018.

18. Story AN. CMS releases BPCI advanced quality measure set. $\mathrm{h}$ ttps://www.accorg/latest-in-cardiology/articles/2019/07/03/15/43/ cms-releases-bpci-advanced-quality-measure-set; 2019.

19. Cortese DA, Korsmo JO. Putting U.S. health care on the right track. N Engl J Med. 2009;361:1326-7.

20. Porter M, Teisberg E. Redefining health care: Creating valuebased competition on results. Boston: Harvard Business School Press; 2006.

21. Gibbons R, Korsmo J, Plutowski R. Payment changes necessary to catalyze higher-quality, lower-cost care. Notre Dame J Law Ethics Public Policy. 2012;25:393-8.

Publisher's Note Springer Nature remains neutral with regard to jurisdictional claims in published maps and institutional affiliations. 